\title{
Synthesis and characterization of electrical conducting chitosan-graft-polyaniline
}

\author{
A. Tiwari ${ }^{*}$, V. Singh ${ }^{2}$ \\ ${ }^{1}$ Division of Engineering Materials, National Physical Laboratory, Dr. K. S. Krishnan Marg, New Delhi, 110 012, India \\ 2Department of Chemistry, University of Allahabad, Allahabad, 211 002, India
}

Received 4 March 2007; accepted in revised form 29 April 2007

\begin{abstract}
A mucopolysaccharide, chitosan was grafted with polyaniline through oxidative-radical copolymerization using ammonium persulfate in acidic medium. The grafting conditions were extensively studied by varying grafting parameters. All the findings have been discussed and proposed a plausible mechanism for the graft copolymerization. The representative chitosan-graft-polyaniline (Ch-g-PANI) was characterized using UV-vis, FTIR, TGA, X-ray diffraction and Scanning electron microscopy taking chitosan as reference. Ch-g-PANI exhibited electrical conductivity, which increases with the extent of grafting onto chitosan backbone. Its electrical conductivity is further influenced by $\mathrm{pH}$ and showed $\mathrm{pH}$ switching electrical conduction behavior when exposed to $\mathrm{NN}_{3} / \mathrm{HCl}$ vapors. The application of conducting biomaterial such as $\mathrm{Ch}-\mathrm{g}$ PANI in the electronic devices especially for the fabrication of sensor devices would be attractive not only in terms of product cost and environmental safety but also from a materials science point of view.
\end{abstract}

Keywords: biopolymers, biocomposites, material testing, thermal properties, industrial applications

\section{Introduction}

Polyaniline (PANI) being most promising organic conducting polymer finds wide applications, such as in rechargeable batteries, corrosion protection of metals, gas-separation membranes, and molecular sensors [1-4]. It bears good environmental, thermal and chemical stability, electrical and optical properties, facile redox and $\mathrm{pH}$-switching behavior [5]. It can be rapidly 'switched' with the addition of acids (protonation) and bases (deprotonation). The acid doped PANI yields conducting emeraldine salt, and is dedoped to form the insulator emeraldine base $[6,7]$. The electrical conductivity of PANI is enhanced by simple change of $\mathrm{pH}$ conditions. Recently, conducting polymers, such as PANI, have been considered for microelectronics applications. The combination of tunable chemical properties with the electronic properties of con-

*Corresponding author, e-mail: ashunpl@gmail.com

(C) BME-PT and GTE ducting polymer has also a tremendous impact on the development of new sensors. The use of conducting polymers in sensors has recently been reviewed [8]. However, PANI has limitations in solubility and mechanical properties [9]. In general, incorporation of PANI into flexible matrix could result in good processability with the electrical conductivity having the requisite properties like chemical stability toward dopants, thermal stability, and insolubility under readily accessible conditions [10, 11]. Chitosan has gained growing interest to prepare semi-interpenetrating chitosan/polyaniline [12-14], because of its excellent film-forming ability, good adhesion, biocompatibility, and high mechanical strength.

Chitosan is a high molecular weight polysaccharide [15] composed of $\beta(1 \rightarrow 4)$ linked 2-deoxy-2amino-D-glucopyranose units and $\beta(1 \rightarrow 4)$ linked 2-deoxy-2-acetamido-D-glucopyranose units. Chi- 
tosan has found potential use in many areas, it is a brittle material and tends to absorb a quantity of moisture and to improve its toughness, solubility and rheological properties, graft copolymerization of a vinyl monomer onto chitosan chains is commonly employed for several applications.

Therefore, chitosan as a low cost, degradable, biocompatible, high molecular weight, branched biopolymer with unique physiochemical properties [16] (presence of $-\mathrm{OH}$ and $-\mathrm{NH}_{2}$ groups offers self doping) is a good candidate to introduce functional groups in PANI, and enhance its selectivity for chemical sensors. In the present study, PANI was grafted on to chitosan in order to synthesize electrically conducting tailor-made material having good $\mathrm{pH}$-switching properties. In the accomplished studies, the reaction mechanism, crystallinty, morphology, thermal and electrical properties were extensively investigated. It was expected that the results would be promising for biomaterial based sensors fabrication and it may be used either in vivo or in vitro.

\section{Experimental section}

\subsection{Materials}

Chitosan (Aldrich; $M_{w} 1.86 \cdot 10^{5}$ ) and aniline (E. Merck) were used after purification. Ammonium persulfate (Aldrich) was used without further purification.

\subsection{Synthesis of PANI}

PANI was synthesized by oxidative polymerization [17] of doubly distilled aniline dissolved in aqueous $\mathrm{HCl}(1 \mathrm{M})$, using ammonium persulfate (APS) in aqueous $\mathrm{HCl}(1 \mathrm{M})$ as an oxidant at $4^{\circ} \mathrm{C}$. Aniline $(0.5 \mathrm{M})$ was dissolved in $200 \mathrm{ml}$ of aqueous solution of $\mathrm{HCl}(1 \mathrm{M})$ and APS $(0.54 \mathrm{M})$ was dissolved in $200 \mathrm{ml} \mathrm{HCl}(1 \mathrm{M})$. The oxidant solution was then added slowly to the aniline solution with continuous stirring at $4^{\circ} \mathrm{C}$. The reaction mixture was kept under continuous stirring for two hours and then kept in a refrigerator overnight. The reaction mixture was filtered and washed with $\mathrm{HCl}(1 \mathrm{M})$ till the filtrate became colorless and then washed with doubly distilled water until the filtrate became acid free. The polymer was ground to a fine powder and low molecular weight oligomers were removed from polyaniline by acetone extraction. The result- ing PANI was dried at $60^{\circ} \mathrm{C}$ in an air oven for $48 \mathrm{~h}$ and stored in a desiccator.

\subsection{Synthesis of Ch-g-PANI}

Chitosan-graft-polyaniline (Ch-g-PANI) was synthesized by a calculated amount of the chitosan dissolved in the minimum or required amount of acidic water in a $150 \mathrm{ml}$ flask. To this solution, a calculated amount of the aniline and hydrochloric acid $(\mathrm{HCl})$ was added and the total volume was made up to $25 \mathrm{ml}$ the flask being thermostated at $25 \pm 0.2^{\circ} \mathrm{C}$ and the contents continuously stirred. After 30 minutes a definite amount of ammonium persulfate was added and this was taken as zero time. Grafting was allowed to proceed for $1 \mathrm{~h}$. The reaction mixture was neutralized by $5 \%$ aqueous $\mathrm{NaOH}$ and the graft copolymer was precipitated with absolute ethanol. The resulting precipitate was washed with N-methyl pyrrolidinone (NMP) in order to separate PANI (homopolymer) from the copolymer. The material was ground to a fine powder and low molecular weight PANI oligomers were removed from Ch-g-PANI via acetone extraction (Figure 1). Finally, the products were dried for 4 days in a vacuum oven at $50^{\circ} \mathrm{C}$.

The percentage and efficiency of grafting were calcalculated by the Equations (1) and (2) [18]:

$\%$ Grafting $[\% \mathrm{G}]=\frac{W_{1}-W_{2}}{W_{0}} \cdot 100$

$\%$ Efficiency $[\% \mathrm{E}]=\frac{W_{1}-W_{2}}{W_{2}} \cdot 100$

where $W_{1}, W_{0}$ and $W_{2}$ denote, respectively, the weights of Ch-g-PANI, chitosan and aniline monomer.

\subsection{Electrical conductivity measurement}

The surface DC electrical conductivity of the Ch-gPANI rectangular pressed pallets (pressure 4 tons $/ \mathrm{cm}^{2}$ ) was measured by the four-probe method [19].

The resistivity can be calculated by Equation (3):

$\rho=2 \pi S\left(\frac{V}{I}\right)$

where, $S$ is the probe spacing [mm], which was kept constant, $I$ is the supplied current in $[\mathrm{mA}]$, and the 


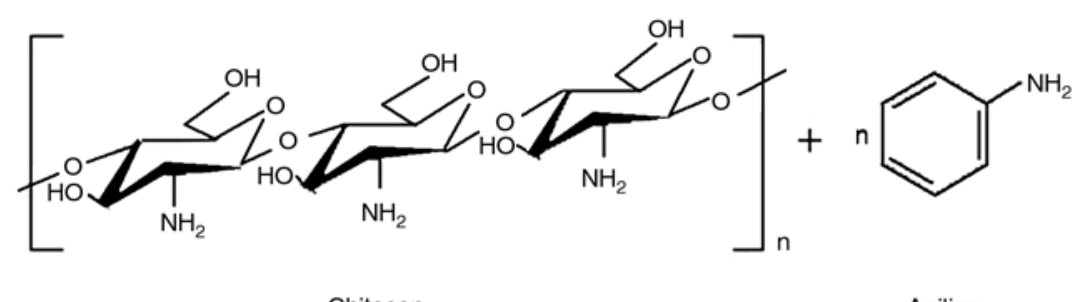

Chitosan

Aniline

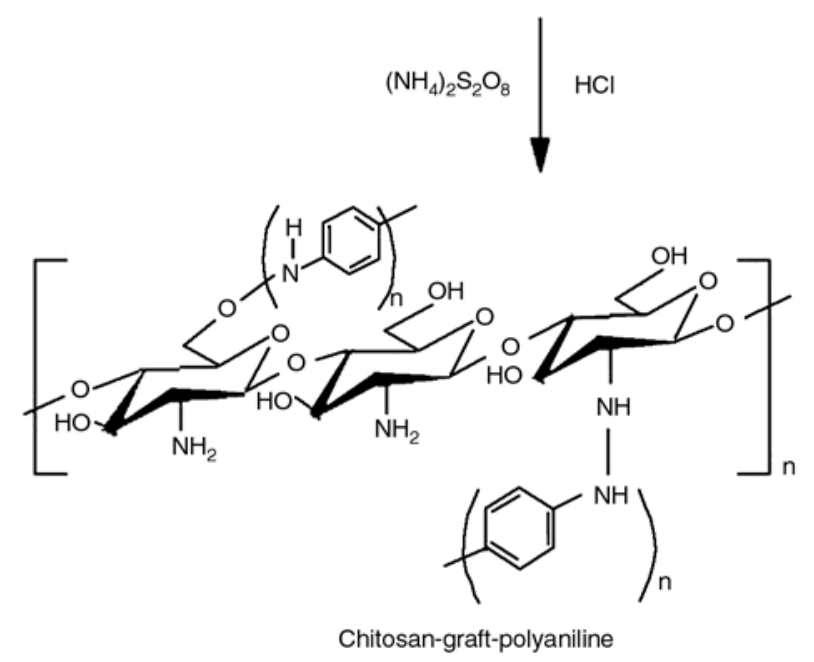

Figure 1. Chitosan-graft-polyaniline synthesized through the oxidative-radical graft copolymerization

corresponding voltage was measured in [mV]. Conductivity can be computed using the Equation (4):

$\sigma=\frac{1}{\rho}$

where $\sigma$ and $\rho$ are conductivity $\left[S \mathrm{~cm}^{-1}\right]$ and resistivity $[\Omega \mathrm{cm}]$ respectively. The electrical conductivity measurements were carried out at intervals of $20 \mathrm{~min}$. The effect of $\mathrm{pH}$ on the electrical conductivity of Ch-g-PANI was studied. All the measurements were repeated three times at intervals of $20 \mathrm{~min}$.

\subsection{Characterization of chitosan-g-PANI}

The UV-vis spectra were recorded on an Ocean Optics HR 4000 High Resolution Spectrophotometer, using aqueous solution of the samples. FTIR spectra were recorded on a Perkin-Elmer instrument (PK11310), using a pellet made with dehydrated $\mathrm{KBr}$ and instrument in the reflectance mode. $\mathrm{X}$-ray diffractogram was taken by Rigaku Rotaflex, RAD/Max-B, Rigaku Corporation, Japan instrument with a scanning speed of $1^{\circ}$ per minute. TGA was recorded on Rigaku Thermoflex, PTC-10A, Rigaku Corporation, Japan with $10^{\circ} \mathrm{C}$ per minute heating rate and Alumina as the reference material. Scanning electron microscope (SEM) was done on a JEOL-840, JEOL Corporation, Japan by making a film of desirable size and coated with gold. The DC electrical conductivity of the samples was measured by making rectangular pressed pallets (pressure 4 tons $/ \mathrm{cm}^{2}$ ) and using the Keithley four-probe system, having a 224 programmable current source, 181 nano voltameter and 195A digital multimeter.

\section{Results and discussion}

\subsection{Determination of optimal grafting conditions}

To optimize the condition for grafting of PANI on to the chitosan, ammonium persulfate/hydrochloric acid/aniline monomer/chitosan concentrations, reaction temperature and reaction time were varied and keeping total volume of the reaction mixture fixed at $25 \mathrm{ml}$. It was observed that $\left(\mathrm{NH}_{4}\right)_{2} \mathrm{~S}_{2} \mathrm{O}_{8} /$ hydrochloric acid system can be efficiently used in the graft copolymerization of polyaniline on to chitosan, where maximum $91.40 \% \mathrm{E}$ could be achieved.

\subsubsection{Effect of ammonium persulfate concentration}

The $\% \mathrm{G}$ increased with an increase in initiator/oxidant concentration, reaching a maximum value at 




Figure 2. Effect of ammonium persulfate concentration at fixed concentration of [aniline] $1.5 \cdot 10^{-2}$, [HCl] 0.3 and [Ch] $1 \mathrm{~g} / 1$ at $25 \pm 0.2^{\circ} \mathrm{C}$ for $1 \mathrm{~h}$

$12.5 \cdot 10^{-2} \mathrm{M}$ of ammonium persulfate (APS) at fixed concentrations of $1.5 \cdot 10^{-2} \mathrm{M}$ aniline, $0.3 \mathrm{M}$ hydrochloric acid and $1 \mathrm{~g} / \mathrm{l}$ Chitosan $(\mathrm{Ch})$ at $25 \pm 0.2^{\circ} \mathrm{C}$ for $1 \mathrm{~h}$ (Figure 2). It was observed that $\% \mathrm{G}$ increased within the APS concentration range from $2.5 \cdot 10^{-2}-12.5 \cdot 10^{-2} \mathrm{M}$. It may be due to the fact that at this concentration range, the produced sulfate ion radical moieties activate the backbone of chitosan and simultaneously produced the PANI ion radical, which takes place immediately followed by the graft copolymerization of the monomer onto the chitosan backbone. Therefore, $\% \mathrm{E}$ increased with an increase in the concentration of persulfate.

\subsubsection{Effect of $\mathrm{HCl}$ concentration}

The effect of acid was studied in the range $0.1-0.5 \mathrm{M}$ at a fixed concentration of $12.5 \cdot 10^{-2} \mathrm{M}$

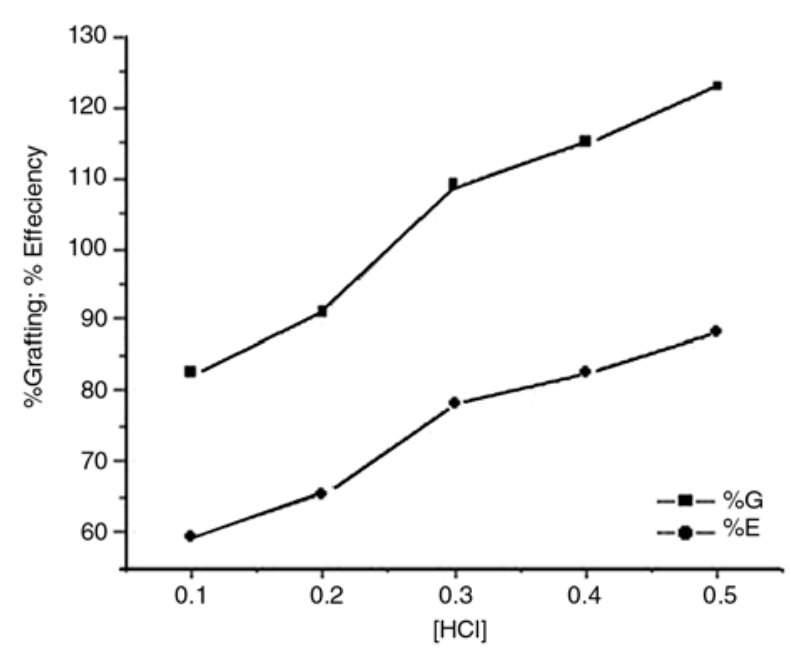

Figure 3. Effect of $\mathrm{HCl}$ concentration at fixed concentration of $\left[\left(\mathrm{NH}_{4}\right)_{2} \mathrm{~S}_{2} \mathrm{O}_{8}\right] 12.5 \cdot 10^{-2}$, [aniline] $1.5 \cdot 10^{-2}$ and $[\mathrm{Ch}] 1 \mathrm{~g} / \mathrm{l}$ at $25 \pm 0 \cdot 2^{\circ} \mathrm{C}$ for $1 \mathrm{~h}$ ammonium persulfate, $1.5 \cdot 10^{-2} \mathrm{M}$ aniline and $1 \mathrm{~g} / \mathrm{l}$ chitosan at $25 \pm 0.2^{\circ} \mathrm{C}$ for $1 \mathrm{~h}$ (Figure 3 ). It was observed that both $\% \mathrm{G}$ and $\% \mathrm{E}$ increases with an increase in the acid concentration, which may be due to a higher degree of protonation of the aniline monomer and acceleration of the propagation of aniline, which can generate more PANI ion radicals.

\subsubsection{Effect of aniline concentration}

The increase in the concentration of monomer from $1 \cdot 10^{-2}-3 \cdot 10^{-2} \mathrm{M}$, resulted in the increase of $\% \mathrm{G}$ and $\% \mathrm{E}$ (up to $1.5 \cdot 10^{-2} \mathrm{M}$ ), under the fixed concentration of $12.5 \cdot 10^{-2} \mathrm{M}$ persulfate, $0.5 \mathrm{M} \mathrm{HCl}$ and $1 \mathrm{~g} / \mathrm{l}$ chitosan at $25 \pm 0.2^{\circ} \mathrm{C}$ for $1 \mathrm{~h}$ (Figure 4). The increase in $\% \mathrm{G}$ and $\% \mathrm{E}$ may be due to the formation of more PANI radical ions, while generating more grafting sites and availability of extra aniline monomer for grafting. With an increasing concentration beyond $1.5 \cdot 10^{-2} \mathrm{M}$ the $\% \mathrm{E}$ decreases slightly, which is probably due to formation of PANI homopolymer.

\subsubsection{Effect of chitosan concentration}

The effect of chitosan concentration was studied in the range of $1-5 \mathrm{~g} / \mathrm{l}$ with the constant concentration of [persulfate] $12.5 \cdot 10^{-2},[\mathrm{HCl}] 0.5$, [aniline] $1.5 \cdot 10^{-2}$ at $25 \pm 0.2^{\circ} \mathrm{C}$ for $1 \mathrm{~h}$ (Figure 5). It was found that the $\% \mathrm{G}$ and $\% \mathrm{E}$ decreased with increasing the concentration of chitosan, which may be due to the increase in the viscosity of the reaction medium causing hindrance in the normal reaction.

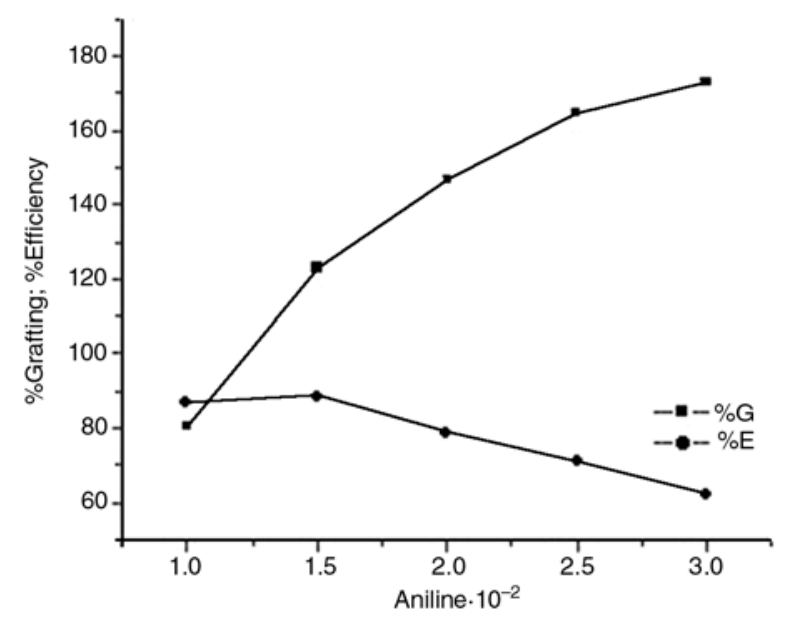

Figure 4. Effect of aniline concentration under the fixed concentration of $\left.\left[\left(\mathrm{NH}_{4}\right)_{2} \mathrm{~S}_{2} \mathrm{O}\right)\right] 1.2 \cdot 10^{-2}$, $[\mathrm{HCl}] 0.5$ and $[\mathrm{Ch}] 1 \mathrm{~g} / \mathrm{l}$ at $25 \pm 0.2^{\circ} \mathrm{C}$ for $1 \mathrm{~h}$ 


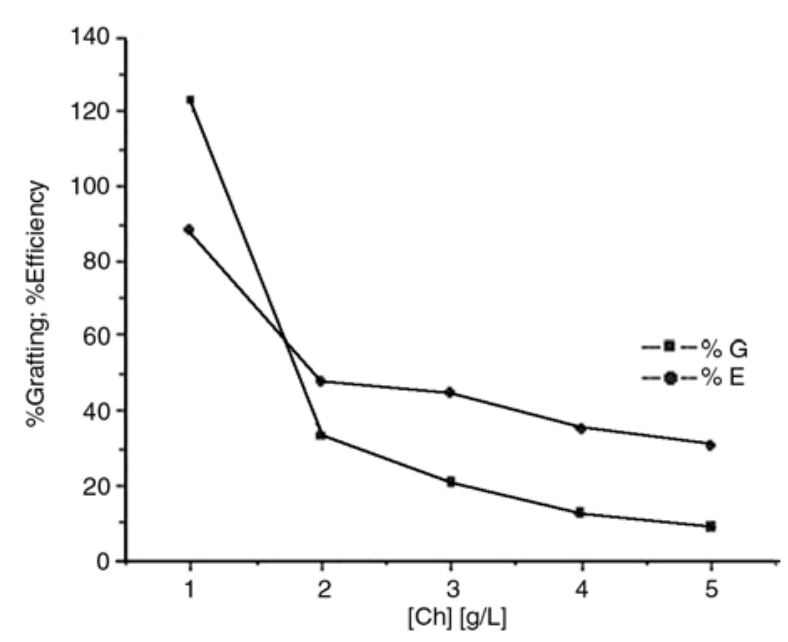

Figure 5. Effect of chitosan concentration with the constant concentration of $\left[\left(\mathrm{NH}_{4}\right)_{2} \mathrm{~S}_{2} \mathrm{O}_{8}\right] 1.2 \cdot 10^{-2}$, [ $\mathrm{HCl}] 0.5$ and [aniline] $1.5 \cdot 10^{-2}$ at $25 \pm 0 \cdot 2^{\circ} \mathrm{C}$ for $1 \mathrm{~h}$

\subsubsection{Effect of temperature}

The grafting reaction was carried out at different temperatures $\left(10-30^{\circ} \mathrm{C}\right)$, while keeping other variables constant; [persulfate] $12.5 \cdot 10^{-2},[\mathrm{HCl}] 0.5$, [aniline] $1.5 \cdot 10^{-2}$ and $[\mathrm{Ch}] 1.0 \mathrm{~g} / \mathrm{l}$ for $2 \mathrm{~h}$ (Figure 6). Maximum $\% \mathrm{G}$ was obtained at $25^{\circ} \mathrm{C}$. The observed increase in $\% \mathrm{G}$ may be attributed to the increase in the number of collisions between the monomer and the chitosan molecules that results in a decrease of the viscosity of the medium at higher temperature. Beyond $25^{\circ} \mathrm{C}, \% \mathrm{G}$, the slight decrease may be due to an increase in ionic mobility of the PANI ion radical and formation of more homopolymer.

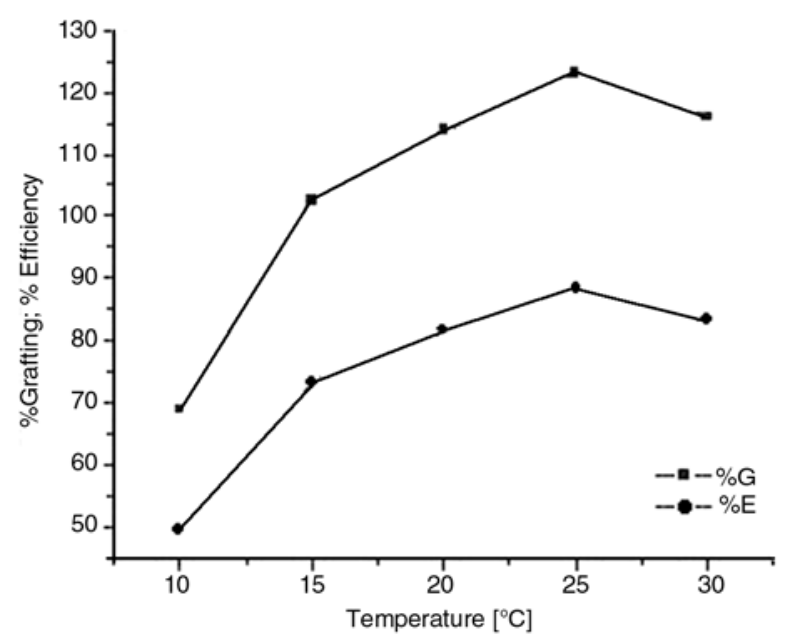

Figure 6. Effect of temperature keeping other variables constant; $\left.\left.\left[\left(\mathrm{NH}_{4}\right)_{2} \mathrm{~S}\right) \mathrm{O}\right)\right] 1.2 \cdot 10^{-2},[\mathrm{HCl}] 0.5$, [aniline] $1.5 \cdot 10^{-2}$ and $[\mathrm{Ch}] 1 \mathrm{~g} / \mathrm{l}$ for $1 \mathrm{~h}$

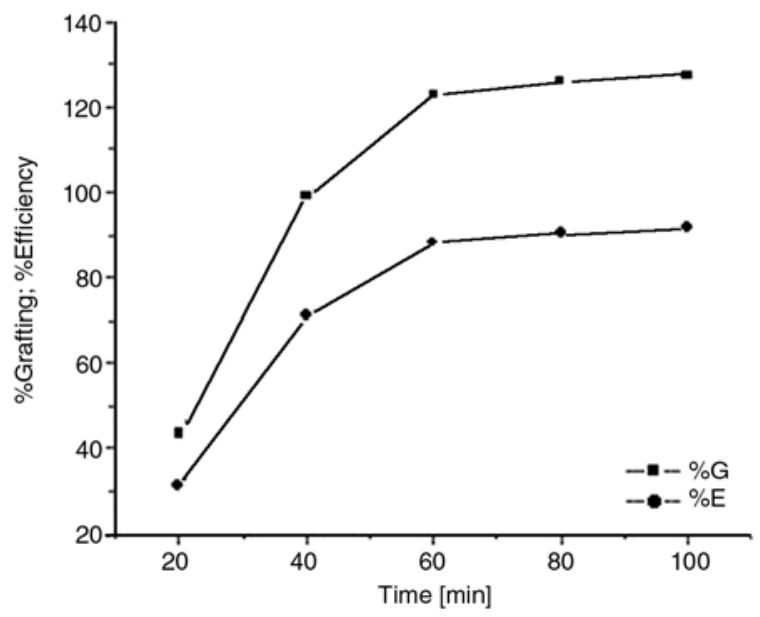

Figure 7. Effect of grafting time keeping other variables constant; $\left[\left(\mathrm{NH}_{4}\right)_{2} \mathrm{~S}_{2} \mathrm{O}_{8}\right] 1.2 \cdot 10^{-2},[\mathrm{HCl}] 0.5$, [aniline] $1.5 \cdot 10^{-2}$ and [Ch] $1 \mathrm{~g} / 1$ at $25 \pm 0.2^{\circ} \mathrm{C}$

\subsubsection{Effect of grafting time}

The grafting reaction was carried out at different times (20-100 min) keeping other variables constant; [persulfate] $12.5 \cdot 10^{-2},[\mathrm{HCl}] 0.5$, [aniline] $1.5 \cdot 10^{-2}$ and $[\mathrm{Ch}] 1.0 \mathrm{~g} / \mathrm{l}$ at $25 \pm 0.2^{\circ} \mathrm{C}$ (Figure 7). Maximum $\% \mathrm{G}$ was obtained at $100 \mathrm{~min}$. The $\% \mathrm{G}$ increased rapidly with increase in time up to 100 min, after which it levels off. Finding could be attributed to a decrease in concentration for both initiator and monomer, thereby a reduction in the number of sites on the backbone accessible for grafting as the reaction procedure.

\subsection{Mechanism for graft copolymerization}

Other researchers [20-22] have exploited free radical copolymerization of vinyl monomers on to chitosan using persulfate. A chain mechanism [23] is involved due to formation of sulphate ion radicals $\left(\mathrm{SO}_{4}^{-}\right)$, which are well-known ion chain carriers for graft copolymerization. At the same time persulfate stimulates the oxidative polymerization reaction of aniline [24] via a medium of cationic radicals and form PANI and PANI radicals. Finally chitosan macro radicals and PANI cation radicals are combined to form Ch-g-PANI graft copolymer. Mechanism may be written as shown by Figure 8 . $\mathrm{SO}_{4}{ }^{-\cdot}$ is the primary radicals, generated from the ammonium persulfate (APS) by the reduction of one electron, expressed as $\mathrm{R}^{\cdot}$ in the Figure 8. Simultaneously, APS generates $\mathrm{SO}_{4}{ }^{-2}$ ions by the reduction of two electrons and act as oxidant. They initiate the oxidative polymerization of aniline, as 


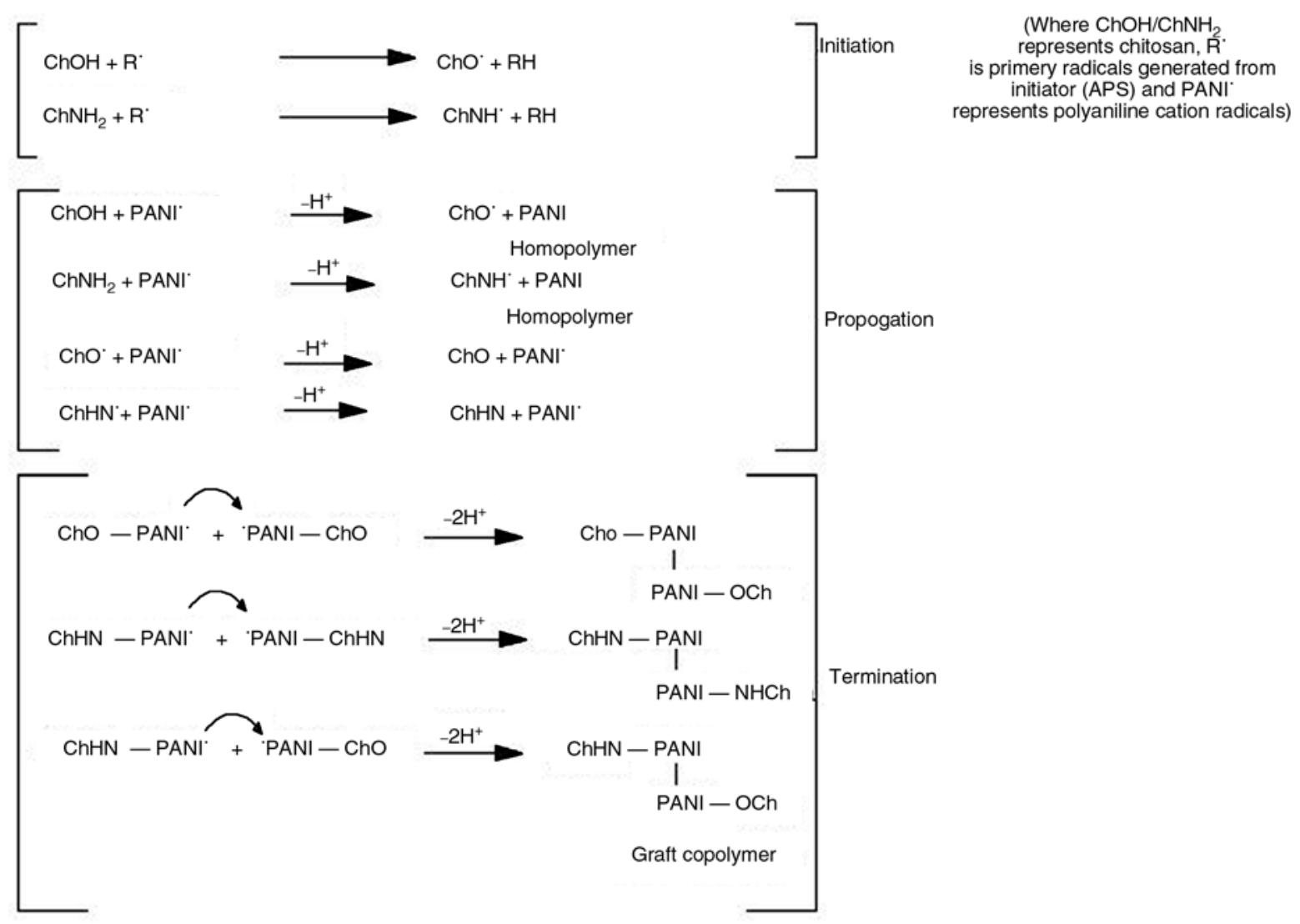

Figure 8. Graft copolymerization of polyaniline on to chitosan

the polymerization of monomer is reported to be faster than the $\mathrm{H}$ abstraction from the biopolymer backbone [25]. The macro radicals $\mathrm{ChO}^{*}$ and $\mathrm{Ch}$ $\mathrm{HN}^{*}$ may be generated by the abstraction of $\mathrm{H}$ by the growing polyaniline ion radical (PANI') in the medium of acid, which may add onto the $\mathrm{ChO}^{\circ}$ and $\mathrm{Ch}-\mathrm{HN}^{\cdot}$ macro radicals generating new radical ChO-PANI ${ }^{\cdot}$ and Ch-NH-PANI and these chains will grow and combined with other ChO-PANI' or Ch-NH-PANI' chains to give a graft copolymer.

\subsection{Characterization of the grafted chitosan}

A representative Ch-g-PANI sample $(173 \% \mathrm{G})$ was characterized by UV-vis, FTIR, XRD, TGA and SEM analysis.

\subsubsection{UV-vis spectra}

The UV-vis spectra of Ch-g-PANI at $\mathrm{pH} 1$ (Figure 9a) showed a broad absorption band at 300-350 nm due to overlapping of glucopyranose components of chitosan and $\pi-\pi^{*}$ transition of benzenoid rings of grafted PANI with bands at $430 \mathrm{~nm}$ (due to polaronic peak of grafted PANI) and at $800 \mathrm{~nm}$ (assigned bipolaronic transitions of grafted

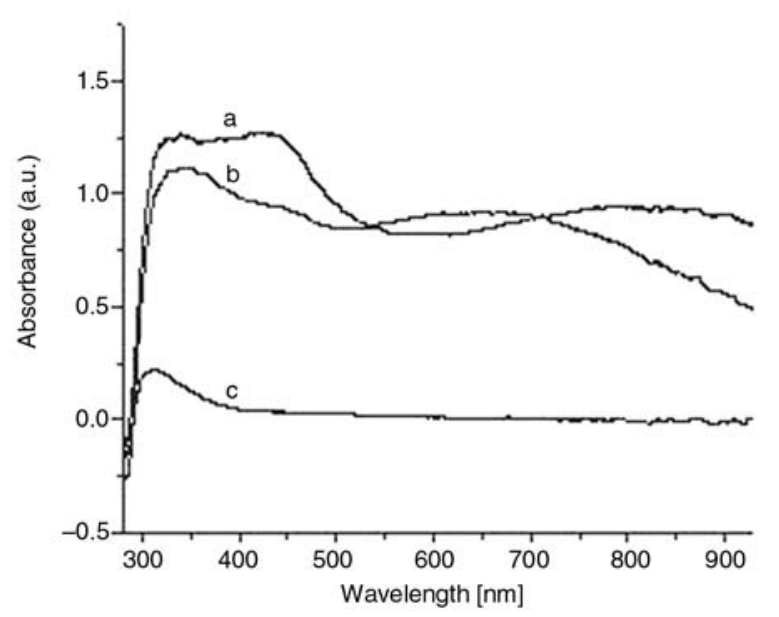

Figure 9. UV-vis spectra of (a) Ch-g-PANI at $\mathrm{pH} 1$, (b) Ch-g-PANI at $\mathrm{pH} 10$, (c) chitosan at $\mathrm{pH} 3$

PANI), while Ch-g-PANI at pH 10 (Figure 9b) clearly showed the dedoping effect (emeraldine base form of grafted PANI) and peak suppressed at $430 \mathrm{~nm}$ but appeared a new peak at $620 \mathrm{~nm}$ (due to the $\pi-\pi^{*}$ transition of quinoid rings on the grafted PANI). These curves (Figure 9a and 9b) indicating that the grafted PANI showed transition 'emeraldine salt-emeraldine base' (protonation-deprotonation or doping-dedoping) and bears emeraldine oxidation state in the media of different $\mathrm{pH}$, which 
affects its electronic absorption spectra [24]. Chitosan showed (Figure 9c) a broad absorption band at $300 \mathrm{~nm}$ (due to glucopyranose components). Furthermore, the characteristric peaks of glucopyranose and PANI was significantly observed and it supports the grafting of PANI on to chitosan. Therefore, UV-vis spectra confirmed the chemical structure, conjugation, electronic transition and doping-dedoping behavior of Ch-g-PANI.

\subsubsection{FTIR spectra}

FTIR spectra of Ch-g-PANI (Figure 10) showed characteristic peaks of PANI as well as chitosan. The infrared band at $3040-3266 \mathrm{~cm}^{-1}$ corresponds to $\mathrm{N}-\mathrm{H}$ stretching with hydrogen bonded 20 amino groups and free $\mathrm{O}-\mathrm{H}$ stretching vibration, $3024 \mathrm{~cm}^{-1}$ corresponds to aromatic $\mathrm{C}-\mathrm{H}$ stretching, 2930 and $2864 \mathrm{~cm}^{-1}$ (due to aliphatic C-H stretching), $1632 \mathrm{~cm}^{-1}$ (due to $\mathrm{C}=\mathrm{O}$ stretching of carbonyl group, typical saccharide absorption), $1526 \mathrm{~cm}^{-1}$ (due to $\mathrm{C}=\mathrm{C}$ stretching of quinoid rings), $1462 \mathrm{~cm}^{-1}$ (due to $\mathrm{C}=\mathrm{C}$ stretching vibration of benzenoid rings), at $1284 \mathrm{~cm}^{-1}$ (due to $\mathrm{C}-\mathrm{N}$ stretching). The absorption band at $1110 \mathrm{~cm}^{-1}$ was assigned to $\mathrm{N}=\mathrm{Q}=\mathrm{N}$ bending vibration shift towards the lower wave number correspond to the PANI. The shift of $1130 \mathrm{~cm}^{-1}$ band to lower wave number could be attributed to the hydrogen bonding between chitosan and imine group of the grafted chain of PANI. The absorption band at $1030 \mathrm{~cm}^{-1}$ (due to $\mathrm{O}-\mathrm{H}$ bending), at $1076 \mathrm{~cm}^{-1}$ (characteristic peaks of a saccharide structure; $\mathrm{C}-\mathrm{O}$

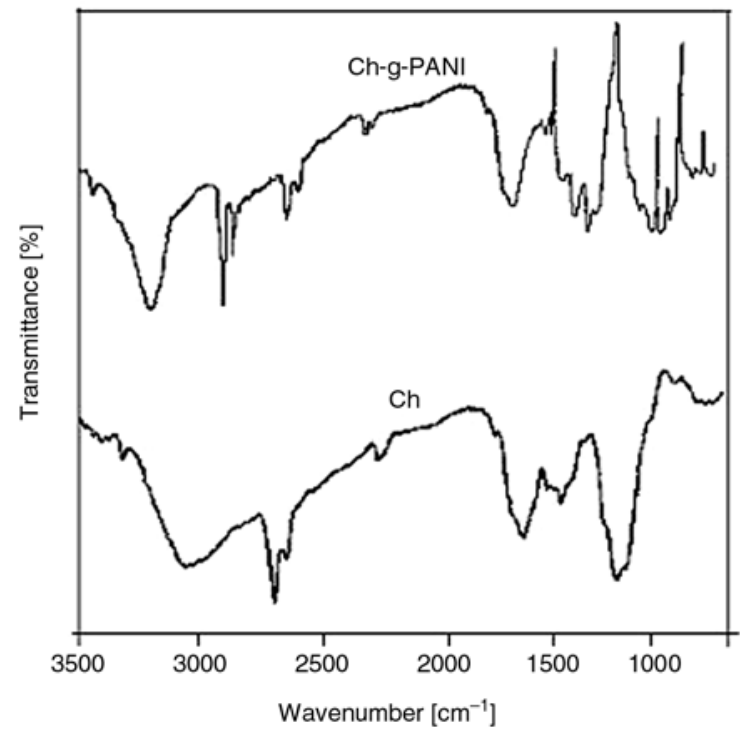

Figure 10. FTIR spectra of Ch-g-PANI and Ch stretching) and at $830 \mathrm{~cm}^{-1}$ was assigned to aromatic $\mathrm{C}-\mathrm{H}$ bending vibration band due to the $1,4-$ disubstituted benzene ring, chitosan showed characteristic peaks of mucopolysaccharide [16]. Hence, FTIR studies clearly suggested the graft copolymerization of polyaniline on to chitosan.

\subsubsection{TGA}

The thermal resistance of Ch-g-PANI was determined by thermogravimetric analysis (TGA) taking chitosan as reference. TGA indicates that the decomposition of the graft copolymer onsets at $176^{\circ} \mathrm{C}$ and $52 \%$ weight loss up to $700^{\circ} \mathrm{C}$ and the graft copolymer slowly degrades, while decomposition onsets of the chitosan was $145^{\circ} \mathrm{C}$ and showed weight loss of $61 \%$ up to $700^{\circ} \mathrm{C}$. It indicates that Ch-g-PANI is more thermally stable than the chitosan (Figure 11).

\subsubsection{XRD spectra}

XRD of the Ch-g-PANI further supported the grafting (Figure 12). XRD spectra of the grafted chitosan showed a crystallinity area in the region of $2 \theta 18-24^{\circ}$ due to the grafting of PANI onto the chitosan backbone, while XRD of the chitosan showed amorphous patterns.

\subsubsection{SEM}

The surface topography of the Ch-g-PANI was studied by scanning electron microscopy (SEM) taking chitosan as reference. The exterior surface of the Ch-g-PANI seems like globules that were accumulated in contrast to the interior structures and showed a porous surface. This fact can be attributed to the porosity of the globular surface in the grafted chitosan, while chitosan showed cotton like accumulation and bears an irregular shape with porous

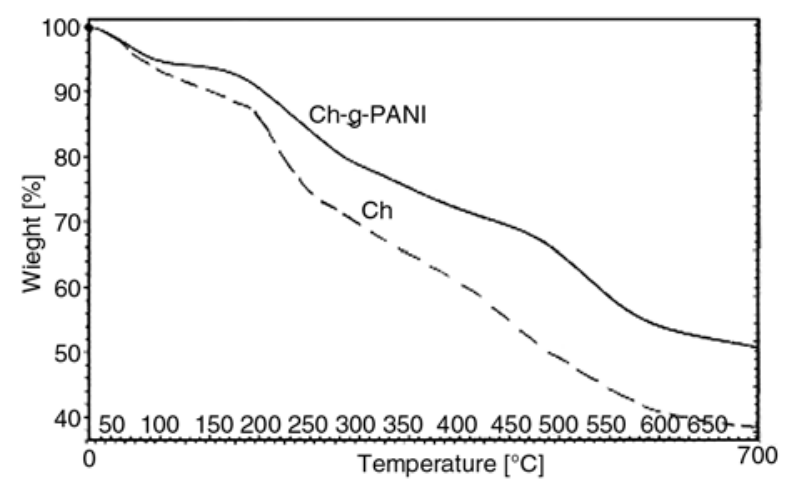

Figure 11. TGA analysis of $\mathrm{Ch}-\mathrm{g}-\mathrm{PANI}$ and $\mathrm{Ch}$ 


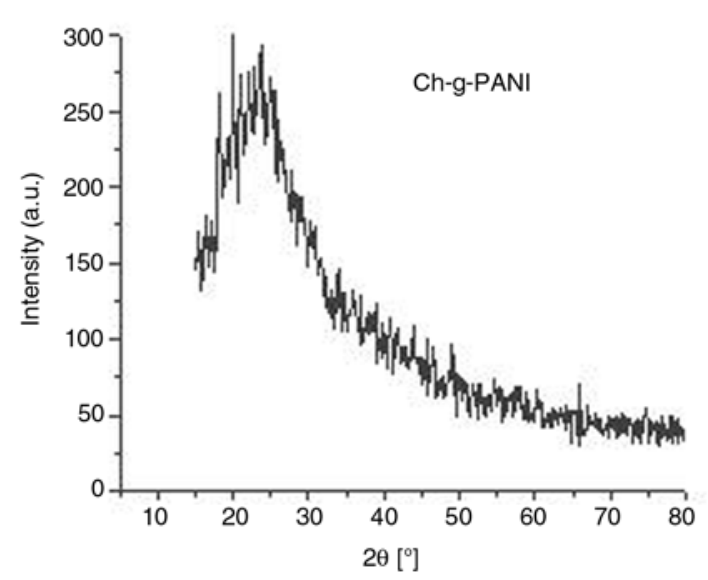

Figure 12. XRD spectra of Ch-g-PANI and Ch
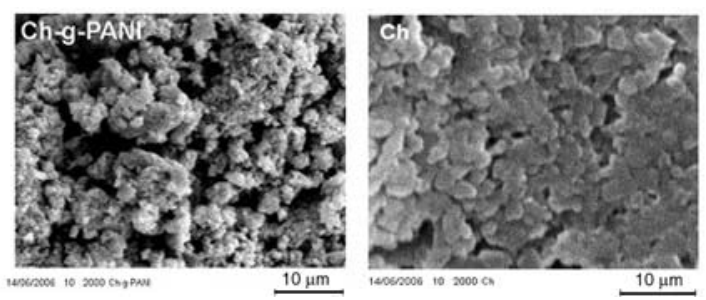

Figure 13. SEM pictures of Ch-g-PANI and $\mathrm{Ch}$

surface. Hence, the surface evidence supports the homogeneity of the uniform grafting of PANI on to the chitosan (Figure 13).

\subsection{Electrical conductivity measurement}

The DC electrical conductivity of grafted (sample of optimum condition; $173 \% \mathrm{G}$ ) was measured at laboratory condition with reference to PANI and chitosan. The results are summarized in the Table 1. The value of DC electrical conductivity was found almost 5000 times higher than that of the chitosan and is attributed to grafting of PANI on to chitosan backbone. The electrical conductivity was influenced with extent of grafting. The results are summarized in the Table 2 . It has been clearly observed that increasing \% grafting, electrical conductivity simultaneously increased. The finding was attributed to the electrical conduction in Ch-g-PANI material, which is purely electronic and occurred due to the grafting of PANI onto chitosan. The electrical conductivity $\mathrm{pH}$ switching behavior of the Ch-g-PANI was measured after doping and dedoping with different concentrations of $\mathrm{HCl}$ and $\mathrm{NH}_{3}$ respectively at a fixed time. The conductivity of the grafted chitosan was increased with increasing dopant concentration (higher at $\mathrm{pH} 0$ and very low at $\mathrm{pH} 10$ ), indicating the formation of emeraldine salt to emeraldine base of the grafted PANI

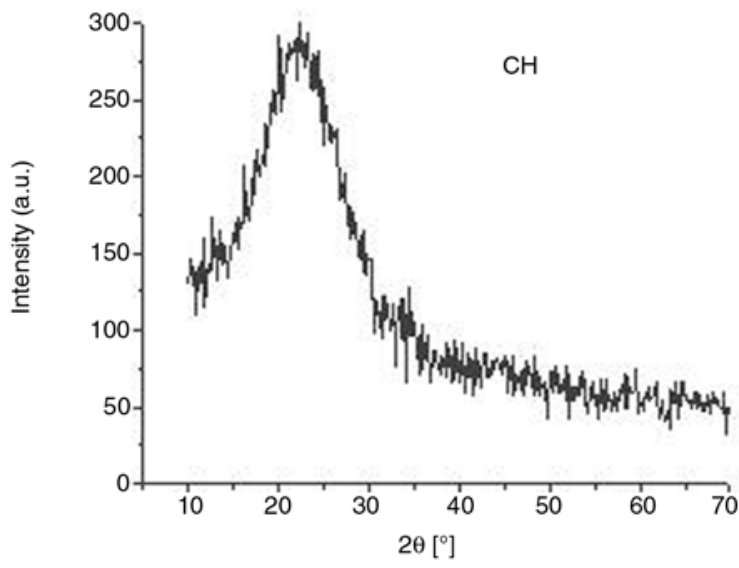

Table 1. Conductivity measurement of polyaniline, chitosan and chitosan-graft-polyaniline

\begin{tabular}{|c|l|c|c|c|}
\hline Sl. No. & \multicolumn{1}{|c|}{ Polymer } & $\mathbf{\% G}$ & $\mathbf{p H}$ & Conductivity $\left[\mathbf{S ~ c m}^{-1}\right]$ \\
\hline 1. & PANI & - & 1 & 0.742 \\
\hline 2. & Chitosan & - & 7 & $9.84 \cdot 10^{-6}$ \\
\hline 3. & Ch-g-PANI & 173 & 1 & $4.03 \cdot 10^{-2}$ \\
\hline
\end{tabular}

Table 2. Effect of \%Grafting on electrical conductivity of chitosan-graft-polyaniline

\begin{tabular}{|c|c|c|c|c|}
\hline Sl. No. & Polymer & $\mathbf{\% G}$ & $\mathbf{p H}$ & Conductivity $\left[\mathbf{S ~ c m}^{-1}\right]$ \\
\hline 1. & Ch-g-PANI & 52.5 & 1 & $6.74 \cdot 10^{-4}$ \\
\hline 2. & Ch-g-PANI & 91 & 1 & $5.19 \cdot 10^{-3}$ \\
\hline 3. & Ch-g-PANI & 146.5 & 1 & $0.96 \cdot 10^{-2}$ \\
\hline 4. & Ch-g-PANI & 173 & 1 & $4.03 \cdot 10^{-2}$ \\
\hline
\end{tabular}

Table 3. Effect of $\mathrm{pH}$ on electrical conductivity of chitosan-graft-polyaniline

\begin{tabular}{|c|c|c|c|c|}
\hline Sl. No. & Polymer & $\mathbf{\% G}$ & $\mathbf{p H}$ & Conductivity $\left[\mathbf{S ~ c m}^{-1}\right]$ \\
\hline 1. & Ch-g-PANI & 173 & 0 & $9.63 \cdot 10^{-2}$ \\
\hline 2. & Ch-g-PANI & 173 & 1 & $4.03 \cdot 10^{-2}$ \\
\hline 3. & Ch-g-PANI & 173 & 3 & $4.91 \cdot 10^{-3}$ \\
\hline 4. & Ch-g-PANI & 173 & 7 & $8.21 \cdot 10^{-4}$ \\
\hline 5. & Ch-g-PANI & 173 & 10 & $0.53 \cdot 10^{-6}$ \\
\hline
\end{tabular}

chain in the material $[26,27]$. All the results are summarized in the Table 3. Therefore, grafted chitosan showed inter-conversion of emeraldine salt to emeraldine base like PANI and hence it could hold promise for sensor applications.

\section{Conclusions}

An inherently anisotropic graft copolymer was synthesized by grafting of polyaniline on to chitosan using persulfate as an initiator in acidic condition. The optimum grafting efficiency was find at $\left[\left(\mathrm{NH}_{4}\right)_{2} \mathrm{~S}_{2} \mathrm{O}_{8}\right] 12.5 \cdot 10^{-2}$, [aniline] $1.5 \cdot 10^{-2}$, [hydro- 
chloric acid] 0.5, [chitosan] $1.0 \mathrm{~g} / \mathrm{l}$, temperature $25 \pm 0.2^{\circ} \mathrm{C}$ and reaction time $100 \mathrm{~min}$. The grafted biomaterial exhibited electrical conductivity with $\mathrm{pH}$ switching behavior like PANI. The electrical conductivity depended on the extent of grafting and $\mathrm{pH}$ of the material. In conclusion, PANI grafted biopolymer will provide good processability with improved solubility, mechanical strength and controlled electrical properties. Therefore, Ch-g-PANI could be exploited for chemical and biosensor applications.

\section{Acknowledgements}

The authors are thankful to the Department of Science and Technology, Govt. of India for generous financial support and to the Director, National Physical Laboratory, New Delhi, India for providing infrastructure facilities to carry out this work.

\section{References}

[1] Sengupta P. P. S., Barik S., Adhikari B.: Polyaniline as a gas-sensor material. Materials and Manufacturing Processes, 21, 263-270 (2006).

[2] Tan C. K., Blackwood D. J.: Interactions between polyaniline and methanol vapour. Sensors and Actuators B: Chemical, 71, 184-191 (2000).

[3] Gosh M., Barman A., Meikap A. K., De S. K., Chatterjee S.: Hopping transport in $\mathrm{HCl}$ doped conducting polyaniline. Physics Letters: A, 260, 138-148 (1999).

[4] Karami H., Mousavi M. F., Shamsipur M.: A new design for dry polyaniline rechargeable batteries. Journal of Power Sources, 117, 255-259 (2003).

[5] Prokes J., Stejskal J.: Polyaniline prepared in the presence of various acids: 2 . Thermal stability of conductivity. Polymer Degradation and Stability, 86, 187195 (2004).

[6] Kahol P. K., Kumar S. K. K., Geetha S., Trivedi D. C.: Effect of dopants on electron localization length in polyaniline. Synthetic Metals, 139, 191-200 (2003).

[7] Kang Y., Kim S. K., Lee C.: Doping of polyaniline by thermal acid-base exchange reaction. Materials Science and Engineering: C, 24, 39-41 (2004).

[8] Adhikari B., Majumdar S.: Polymers in sensor applications. Progress in Polymer Science, 29, 699-766 (2004).

[9] Tiwari A.: Gum arabic-graft-polyaniline: An electrically active redox biomaterial for sensor applications. Journal of Macromolecular Science Part A: Pure and Applied Chemistry, 44, 735-745 (2007).

[10] Lu X., Tan C. Y., Xu J., He C.: Thermal degradation of electrical conductivity of polyacrylic acid doped polyaniline: effect of molecular weight of the dopants. Synthetic Metals, 138, 429-440 (2003).
[11] Takahashi K., Nakamura K., Yamaguchi T., Komura T., Ito S., Aizawa R., Murata K.: Characterization of water-soluble externally $\mathrm{HCl}$-doped conducting polyaniline. Synthetic Metals, 128, 27-33 (2002).

[12] Shin S. R., Park S. J., Yoon S. G., Spinks G. M., Kim S. I., Kim S. J.: Synthesis of conducting polyaniline in semi-IPN based on chitosan. Synthetic Metals, 154, 213-216 (2005).

[13] Kim S. J., Shin S. R., Spinks G. M., Kim Y., Kim S. I.: Synthesis and characteristics of a semi-interpenetrating polymer network based on chitosan/polyaniline under different ph conditions. Journal of Applied Polymer Science, 96, 867-873 (2005).

[14] Peniche C., Argüelles-Monal W., Davidenko N., Sastre R., Gallardo A., Roman S. J.: Self-curing membranes of chitosan/PAA IPNs obtained by radical polymerization: preparation, characterization and interpolymer complexation. Biomaterials, 20, 1869 1878 (1999).

[15] Singh V., Tiwari A., Tripathi D. N., Sanghi R.: Microwave assisted synthesis of chitosan-graft-polyacrylamide: an efficient metal ion binder. Polymer, 47, 254-260 (2006).

[16] Singh V., Tripathi D. N., Tiwari A., Sanghi R.: Microwave Promoted Synthesis of Chitosan-graftedpoly(acrylonitrile). Journal of Applied Polymer Science, 95, 820-825 (2004).

[17] Albuquerque J. E., Mattoso L. H. C., Balogh D. T., Faria R. M., Masters J. G., MacDiarmid A. G.: A simple method to estimate the oxidation state of polyanilines. Synthetic Metals, 113, 19-22 (2000).

[18] Singh V., Tiwari A., Tripathi D. N., Sanghi R.: Microwave assisted synthesis of Guar-g-poly(acrylamide). Carbohydrate Polymers, 58, 1-6 (2004).

[19] Tiwari A., Mishra A. P., Dhakate S. R., Khan R., Shukla S. K.: Synthesis of electrically active biopolymer-SiO2 nanocomposite aerogel. Materials Letters, Doi: 10.1016/j.matlet.2007.02.076 (2007).

[20] Singh V., Tiwari A., Singh S. P., Shukla P. K., Sanghi R.: Co-polymerization of methylmethacrylate on to galactomannans using $\mathrm{K}_{2} \mathrm{~S}_{2} \mathrm{O}_{8}$ /ascorbic acid redox system. Reactive and Functional Polymer, 66, 13061318 (2006).

[21] Singh V., Tiwari A., Sanghi R.: Studies on $\mathrm{K}_{2} \mathrm{~S}_{2} \mathrm{O}_{8}$ / ascorbic acid initiated synthesis of Ipomoea dasysperma seed gum-g-poly(acrylonitrile): A potential industrial gum. Journal of Applied Polymer Science, 98, 1652-1662 (2005).

[22] Singh V., Tiwari A., Tripathi D. N., Sanghi R.: Poly(acrylonitrile) grafted ipomoea seed-gums: A renewable reservoir to industrial gums. Biomacromolecules, 6, 453-456 (2005).

[23] Singh V., Tiwari A., Pandey S., Singh S. K.: Microwave promoted synthesis of starch-graftpoly(acrylonitrile). Express Polymer Letters, 1, 51-58 (2007). 
[24] Gospodinova N., Terlemezyan L.: Conducting polymers prepared by oxidative polymerization: Polyaniline. Progress in Polymer Science, 23, 1443-1484 (1998).

[25] Singh V., Tiwari A., Tripathi D. N., Sanghi R.: Grafting of polyacrylonitrile on to guar gum under microwave irradiation. Journal of Applied Polymer Science, 92, 1569-1575 (2004).
[26] Luthra V., Singh R., Gupta S. K., Mansingh A.: Mechanism of dc conduction in polyaniline doped with sulfuric acid. Current Applied Physics, 3, 219-222 (2003).

[27] Matsuguchi M., Io J., Sugiyama G., Sakai Y.: Effect of $\mathrm{NH}_{3}$ gas on the electrical conductivity of polyaniline blend films. Synthetic Metals, 128, 15-19 (2002). 